\title{
Role of Natural Killer Cells in Lung Cancer
}

\begin{abstract}
Purpose

One of the key immune cells involved in the pathogenesis of lung cancer is natural killer (NK) cells and these cells are novel targets for therapeutic applications in lung cancer. The purpose of this review is to summarize the current literature on lung cancer pathogenesis with a focus on the interaction between NK cells and smoking, how these factors are related to the pathogenesis of lung cancer and how NK cell based immunotherapy effect lung cancer survival.
\end{abstract}

\section{Methods}

The relevant literature from PubMed and Medline databases is reviewed in this article.

\section{Results}

The cytolytic potential of NK cells are reduced in lung cancer and increasing evidence suggests that improving NK cell functioning may induce tumour regression. Recent clinical trials on NK cell based novel therapies such as cytokines including interleukin (IL)-15, IL-12 and IL-2, NK92 cell lines and allogenic NK cell immunotherapy showed promising results with less adverse effects on the lung cancer survival.

\section{Conclusions}

The NK cell targeting strategy has not yet been appproved for lung cancer treatment. More clinical studies focusing on the role of NK cells in lung cancer pathogenesis are warranted to develop novel NK cell based therapeutic approaches for the treatment of lung cancer.

\section{Keywords:Immunotherapy; Lung cancer; Natural killer cells; Non-small cell lung cancer; Small cell lung cancer}

\section{Introduction}

Cancer is one of the leading causes of morbidity and mortality worldwide, with approximately 14 million new cases and 8.2 million cancer deaths reported in $2012^{1}$. Lung cancer represents $13.2 \%$ of all newly diagnosed cancers in the US and the estimated number of deaths from pulmonary malignancies is 155,870 in 2017 , showing the importance of implementing prevention and treatment strategies for this mortal disease ${ }^{2}$. Former or current status of smoking is the main risk factor for the majority of diagnosed lung cancers ${ }^{3}$. Due to 
high smoking prevalence in Turkey, the incidence of lung cancer diagnosed in males (98 $/ 100,000)$ is almost two times higher than the incidence in the US $(52.5 / 100,000)^{4}$.

Lung cancer mainly consists of two subtypes, small cell lung cancer (SCLC) and nonsmall cell lung cancer (NSCLC) and standard anti-cancer therapy includes chemotherapy, radiotherapy and/or surgery ${ }^{1}$. Prognosis of lung cancer depends on the staging and histological classification of the tumor as well as clinical factors. According to the report of Surveillance, Epidemiology, and End Results Program (SEER), National Institute of Health (NIH), only $18.1 \%$ of patients with lung cancer have survived 5 or more years after the diagnosis ${ }^{2}$. Various genetic mutations were reported in both SCLCs and NSCLCs. Recently, treatment strategies to target these mutations including activating mutations of epidermal growth factor receptor (EGFR) and anaplastic lymphoma kinase (ALK) translocations with encouraging results led these treatments to become incorporated into standard treatment algorithms ${ }^{6}$. Additionally, the interaction between the immune system and lung cancer has also been studied for novel therapeutic strategies in lung cancer management such as cancer immunotherapy.

The growing understanding of immune mechanisms in lung cancer indicates highly complex immune interactions in each stage of lung cancer. One of the key immune cells in the tumor microenvironment is Natural Killer (NK) cells which can play an important role in initiating and promoting cancer. They are effective at the first line defence for tumor elimination and major functions include cytotoxicity and cytokine production which help to kill tumor cells ${ }^{7}$ . It was reported that peripheral NK cell cytotoxicity and interferon gamma (INF- $\gamma$ ) production was reduced in lung cancer patients ${ }^{8-10}$. Furthermore, infiltration of the tumor tissue by NK cells was associated with good prognosis in lung cancer ${ }^{11}$. However, there is still limited data regarding the effects of NK cells on lung cancer pathogenesis. We aim to provide a literature review on lung cancer pathogenesis with a focus on the role of NK cells in lung cancer development. The interaction between NK cells and smoking, how these factors are related to the pathogenesis of lung cancer and NK cell-based immunotherapy will also be discussed.

\section{NK cells}

NK cells constitute $10 \%$ of peripheral lymphocytes in human. They are present at a high frequency in the peripheral blood, lymph nodes, spleen, and bone marrow ${ }^{12,13}$ and they can also migrate to inflammation sites due to different chemoattractants ${ }^{13}$. NK cells are important in both innate and adaptive immune system. They stimulate maturation of dendritic cells, and play a role in activation of monocytes and cytotoxic T cells ${ }^{7}$. In addition, cytokines produced by NK 
cells influence T helper $\left(\mathrm{T}_{\mathrm{H}}\right)$ cell polarization, and activated NK cells either stimulate or inhibit B cells to produce immunoglobulin ${ }^{7}$. NK cell markers are CD16 and CD56 which help the identification of NK cell subsets ${ }^{14}$. Majority of human NK cells have low expressions of density expression of CD56 (CD56dim), and express high levels of CD16 (CD16bright). Another type of NK cell is called CD56bright which is further classified as CD16+ and CD16- subtypes. These 2 subsets of NK cells have different levels of cytotoxicity and cytokine production. CD56bright cells are the major subset in the secondary lymphoid organs, and can produce large amounts of cytokines. In contrast, CD56dim cells possess a strong cytolytic capacity, and secrete low levels of cytokines ${ }^{7,15}$.

The major functions of NK cells are cytotoxicity and cytokine production ${ }^{7}$. Activated NK cells produce various cytokines, including IFN- $\gamma$, tumor necrosis factor alpha (TNF- $\alpha$ ) and granulocyte-macrophage colony-stimulating factor $(\mathrm{GM}-\mathrm{CSF})^{16}$. IFN- $\gamma$ maintains anti-tumor activity by inducing MHC I expression and activating CD8+ T-cells to kill tumor cells ${ }^{17}$. TNF$\alpha$ can have direct cytotoxic activity by triggering caspase 8 -mediated apoptosis ${ }^{18}$. NK cell mediated lysis of the target cell occurs by the release of cytotoxic molecules perforin and granzymes A and $\mathrm{B}^{7}$. Cytotoxicity of NK cells can be explained through 2 mechanisms: natural cytotoxicity against tumor cells or virally infected cells in the absence of prior stimulation, and antibody-dependent cellular cytotoxicity against IgG-coated target cells ${ }^{7}$. NK cells express a variety of receptors such as activating receptors NKG2D; the natural killing receptors NKp30, NKp44, NKp46, and NKp80; the SLAM-family receptors and killer Ig-like receptors (KIR) families that transmit inhibitory signals when engaged by MHC I molecules expressed by target cells ${ }^{17}$. Among various NK cell receptors, NKG2D receptor is unique since it recognizes antigens frequently expressed on different tumors, indicating the role of NK cells in tumor immune surveillance ${ }^{19}$. Although NKG2D receptor-ligand interaction is effective to kill tumor cells, NK cells are also capable of destroying certain tumors which lacks ligands for NKG2D receptor. NKp30, NKp44 and NKp46 receptors linked to Immunoreceptor Tyrosine-based Activating Motif (ITAM)-bearing signaling adaptor molecules were proposed to be responsible for the recognition and destruction of tumors lacking NKG2D ligands ${ }^{20}$. KIR expression by NK cells also allows for recognition and elimination of tumor cells which lost class I MHC expression $^{17}$.

\subsection{NK cells and Smoking}

Cigarette smoking is a predominant etiologic factor for lung cancer ${ }^{3}$ and the effect of cigarette smoking on immune cells has been investigated for decades. It has already been 
revealed that cigarette smoking impairs the immune defense ${ }^{21}$. In murine lung metastasis model, increased melanoma metastasis to the lungs was associated with exposure to cigarette smoke. By using RAG2 ${ }^{-/-}$( $\mathrm{T}$ and $\mathrm{B}$ cell deficient) and RAG2 $2^{-/-} \gamma_{\mathrm{c}}{ }^{-/-}(\mathrm{NK}$ cell, B and T cell deficient) mice, destructive effect of cigarette smoking on tumor immune surveillance were shown to be resulted from weakened NK cell activity. Cigarette smoking suppresses NK cell activation and NK cell dependent tumor immune surveillance ${ }^{22}$. In murine chronic obstructive lung disease (COPD) model, NK cell functioning following smoke exposure was examined and smoke exposure was found to promote the expression of IFN- $\gamma$ and CD107a in NK cells upon stimulation, and enhanced NK cell responses ${ }^{23}$. Spleen cells from monkeys exposed to high dose (human equivalent of 3 packs/day) cigarette smoke had a significant reduction in their NK cell-mediated lytic activity and a decreased response to concanavalin $\mathrm{A}^{24}$.

Previous observations on smoking-associated NK cell dysfunction were supported by in vitro studies. Cigarette smoke was reported to inhibit IFN- $\gamma$ and TNF- $\alpha$ in human NK cells from non-smokers in vitro. Reduced cytotoxic activity in NK cells was also observed in smokers $^{25}$. Cigarette smoke suppresses the induction of IL-15 and IL-15 mediated NK cell functions in human peripheral blood mononuclear cells (PBMCs) ${ }^{26}$. Exposure of PBMCs from non-smoking individuals to smoke-conditioned media is associated with defective activation of IRF-3 and NF-kB in PBMCs ${ }^{27}$. Exposure of PBMCs to cigarette smoke also was also found to be related with impaired NK cell cytolytic potential to kill K562 cancer cells through inhibition of IL-15 28 . In previous human studies, smoking caused a decrease in serum immunoglobulin concentrations together with a reduction of the absolute number of CD16+ NK-cells, especially in men smoking for more than ten years ${ }^{29}$. Another study investigating several immunological markers in pairs of healthy smoking and non-smoking monozygotic twins who shared similar environmental conditions and lifestyles demonstrated that smoking was associated with significant increases in the number of circulating leukocytes, neutrophils, T helper cells and B cells, and decreases in the number of NK cells and CD8+ T cells ${ }^{30}$. A study by Luppi et al. reported that the frequency of circulating NK cells was significantly decreased in early pregnancy of women who were light or heavy smokers compared to nonsmoker pregnant women $^{31}$.

\subsection{NK cells and Lung Cancer}

NK cells produce cytolytic enzymes and cytokines, including granzyme B, perforin and IFN- $\gamma$ which are essential to inhibit proliferation of lung cancer cells ${ }^{32}$. There is significantly reduced granzyme B production by NK cells from cancer tissue obtained by lung resection 
surgery when compared to non-cancer tissue ${ }^{33}$. Tumor cells also release immunosuppressive cytokines such as IL-10 or TGF- $\beta$ which suppress NK cell function ${ }^{33-37}$. TGF $\beta 1$ has been shown to inhibit human NK-mediated antitumor cytotoxicity by selectively down-regulating the surface expression of NKp30 and NKG2D ${ }^{33}$. The expression of TGF- $\beta 1$ protein in NSCLC tissue was significantly higher than in the normal lung tissue ${ }^{34}$. IL-10 production was also increased in NSCLC tissue and an increased serum IL-10 concentration was correlated with reduced survival rate ${ }^{35,36}$. IL-10 is mainly produced by regulatory T cells (Treg) and increased proportions of Tregs and decreased proportions of NK cells in tumor tissue have been reported in lung cancer ${ }^{37}$. There are specific molecules expressed on NK cells which can play a role in malignancies including lung cancers. $\mathrm{T}$ cell immunoglobulin- and mucin-domain-containing molecule-3 (Tim-3) is expressed on NK cells and plays an important role in regulating NK-cell functions in cancer $^{38}$. Higher Tim-3 expression in CD3-CD56+ NK cells and CD3-CD56dim NK-cell subset was found to be associated with decreased NK cell cytotoxicity and poor prognosis in lung adenocarcinoma ${ }^{39}$. Futhermore, mRNA levels of the two major NK cell receptors, NCR1/NKp46 and NCR3/NKp30 were reduced in NSCLC which was corraleted with poor overall and progression-free survival ${ }^{40}$. The percentage of peripheral blood NK cells expressing the activating receptor NKp46 was significantly reduced in patients with NSCLC or SCLC compared to healthy donors ${ }^{9}$. Aforementioned studies underscore the role and importance of NK cells in the development of lung cancer and the effect of NK cells on the prognosis of and survival from this disease.

The survival of lung cancer patients was positively correlated with the degree of NK cell infiltration in lung cancer ${ }^{41}$. For instance, there was a significant correlation between survival rates and the number of tumor infiltrating NK cells in patients with primary squamous cell lung carcinoma ${ }^{11}$. Intratumoral NK functionality is essential in addition to the quantity of tumor infiltrating NK cells in the formation of immunosuppressive tumor microenvironment. Tumor microenvironment has been demonstrated to impair NK cell function locally, rendering them less tumoricidal. Platonova et al. reported that intratumoral NK cells had a decreased expression of a cluster of NKp30, NKp80, DNAM-1, CD16, and ILT2 receptors as compared to the NK cells from distal lung tissues or blood from the same patients or healthy donors ${ }^{8}$. Moreover, intratumoral NK cells were defective in the ability to activate degranulation and IFN- $\gamma$ production ${ }^{8}$. In another study, CD56brightCD16- NK cell subset was mainly observed in tumor infiltrate. The cytolytic potential of these cells isolated from cancer tissues was lower than that of NK cells from peripheral blood or normal lung tissue ${ }^{42}$. Factors that may cause 
decreased or dysfunctional NK cell activity in the tumor microenvironment are yet to be discovered. Interestingly, Esendagli et al. found that non-malignant tissue areas in NSCLC were also infiltrated by NK cells which shows strong cytotoxic activity ex vivo ${ }^{43}$. Data coming from multiple studies may prove the protective role of NK cells in and around the tumor microenvironment.

\section{NK cell-based immunotherapy in lung cancer}

The influence of NK cells in the formation of immunosuppressive tumor microenvironment makes these cells a target for the development of treatments in cancer. These cells are increasingly considered as immunotherapeutic agents for the patiens with different malignancies. Recent studies have been focused on proliferation of NK cell population, inhibition of NK cell suppression mechanisms and enhancement of NK cell target recognition ${ }^{44}$. For instance, NK cell-mediated antibody-dependent cell-mediated cytotoxicity could be enhanced by using monoclonal antibodies with cytokines or with drugs that prevent both CD16 and NKG2D ligands shedding on tumor cells $\mathrm{s}^{45-47}$. Up to date, most of studies regarding NK cell based adoptive immune therapy have performed in hematologic malignancies. However, there have been increasing data showing that NK cells can selectively recognize and kill cancer stem cells in solid tumors ${ }^{48}$. In 2000 , Kim et al. showed the fundamental role of NK cells in prevention of lung metastasis ${ }^{49}$. In the study of Tonn et al., the NK-92 cell line (CD56+/CD3-) was isolated from a patient with lymphoma, expanded with recombinant interleukin-2 and infused to patients with solid tumors/sarcomas. After the treatment, an anti-tumor effect was observed in $75 \%$ of the patients with lung cancer ${ }^{50}$. Additionally, Hiraki et al. demonstrated that IL-12 activation of peripheral blood mononuclear cells can increase the cytolytic activity of lung cancer cells when combined with IL- ${ }^{51}$. Furthermore, IL-15 is another theuropatic target which is a homeostatic factor and required for NK cell development. Recently, a phase I open label, non-randomized dose escalation study of subcutaneous rhIL-15 was conducted in refractory solid tumor cancer patients including advanced metastatic melanoma, renal cell carcinoma, NSCLC and squamous cell head and neck carcinoma ${ }^{52}$. Subcutaneous rhIL-15 treatment produced substantial increases in circulating NK and CD8+ T cells which can prove the effectiveness of immunotherapies targeting NK cells and lead to more studies on the immunotherapy agents for different malignancies including lung cancer.

In a murine lung cancermodel, Cui et al. revealed that mixed adoptive immunotherapy with NK and dendritic cells promoted NK cell activity without exogenous cytokines and this data may prove to be useful in lung cancer treatment ${ }^{53}$. Additionally, Zhang et al. studied the 
efficacy of adaptive transfer of NK and cytotoxic T lymphocytes mixed effector cells in NSCLC patients $^{54}$. A prolonged overall survival was shown in patients after receiving NK cell based immunotherapy. In a recent study of Lin et al., the clinical outcomes of cryosurgery combined with allogenic NK cell immunotherapy for the treatment of advanced NSCLC were improved with enhanced immune functions and quality of life ${ }^{55}$. Krause et al. investigated the effectiveness of autologous transfer of NK cells activated ex vivo by the 14 amino acid sequence (TKD) of heat shock protein 70 (Hsp70) plus low-dose IL-2 in one NSCLC patient and 11 colorectal cancer patients who had failed standard therapies ${ }^{56}$. An increased lytic activity against Hsp70 membrane-positive tumor target cells was shown in 10 out of 12 patients with no severe toxicity after NK cell reinfusion. Moreover, an ongoing phase II randomized control trial has been recently testing the efficacy of targeted NK cell based adoptive immunotherapy for the treatment of 90 patients with stage IIIA/B NSCLC after radiochemotherapy (NCT02118415) $^{57}$. Efficacy of targeted NK cell based adoptive immunotherapy was also tested in SCLC patients. Ding et al. investigated the efficacy and safety of cellular immunotherapy with autologous NK, $\gamma \delta \mathrm{T}$, and cytokine-induced killer cells as maintenance therapy for 29 SCLC patients ${ }^{58}$. Immunotherapy as the maintance therapy prolonged the survival of patients with SCLC. In fact, the great majority of NK-based lung cancer treatment mechanisms were identified in murine models. There are a limited number of clinical and/or phase studies in the literature, summarized in Table 1. However, NK cells remain to have a promising therapeutic potential for lung malignancies.

\section{Conclusion}

NK cells have been shown to play an important role in the control of effective immune responses against lung cancer. NK cells can kill cancer cells via direct cytotoxity, induction of apoptosis or IFN- $\gamma$ secretion. Cigarette smoking suppresses NK cell activation and NK cell dependent tumor immune surveillance. Majority of studies investigating NK cells in animal cancer models concluded that NK cells can play an anti-neoplastic role in lung cancer. Increasing evidence suggests that improving NK cell functioning may induce tumour regression. Clinical trials on NK based novel therapies such as cytokines, NK-92 cell lines, allogenic NK cell immunoteherapy have showed increasedsurvival rates from lung cancer. However, current clinical evidence is not sufficient to support the use of these therapies for thetreatment of lung cancer. More clinical studies focusing on the role of NK cells in lung carcinogenesis are warranted to develop novel NK-based therapeutic approaches for lung 
cancer. Such immunological studies using NK cell-based approaches may change the structure and rules of lung cancer treatment in the future.

Table 1: Clinical studies evaluating the NK cell-based immunotherapy in lung cancer

\begin{tabular}{|l|l|l|l|}
\hline Drug & Effect on NK cells & Cancer type & Stage \\
\hline $\begin{array}{l}\text { Cytokines } \\
\text { IL-12 combined with IL-2 }\end{array}$ & $\uparrow$ Cytotoxicity & NSCLC & Phase 1 \\
\hline NK-92 cell line (aNK) & $\uparrow$ Cytotoxicity & $\begin{array}{l}\text { NSCLC } \\
\text { SCLC }\end{array}$ & \\
\hline $\begin{array}{l}\text { Adaptive transfer of NK cells } \\
\text { Allogenic NK cell immunotherapy }\end{array}$ & $\uparrow$ Cytotoxicity & NSCLC & Phase 1,2 \\
& & SCLC & \\
\hline
\end{tabular}

\section{References:}

1. World Health Organization. International Agency for Research on Cancer. GLOBOCAN 2012: Estimated cancer incidence, mortality and prevalence worldwide in 2012 http://globocan.iarc.fr/Pages/fact_sheets_cancer.aspx (last accessed November 2017).

2. National Cancer Institute. Surveillance, Epidemiology and End Results Programme. Cancer Stat Facts: Lung and Bronchus Cancer. https://seer.cancer.gov/statfacts/html/lungb.html (last accessed November 2017).

3. IARC Working Group on the Evaluation of Carcinogenic Risks to Humans. Tobacco smoke and involuntary smoking. IARC Monogr Eval Carcinog Risks Hum 2004;83:1-1438.

4. Anton-Culver H, Chang J, Bray F, Znaor A, Stevens L, Eser S, et al. Cancer burden in four countries of the Middle East Cancer Consortium (Cyprus; Jordan; Israel; Izmir (Turkey) with comparison 
to the United States surveillance; epidemiology and end results program. Cancer Epidemiol 2016;44:195-202.

5. Felip E, Stahel RA, Pavlidis N; ESMO Guidelines Task Force. ESMO Minimum Clinical Recommendations for diagnosis, treatment and follow-up of non-small-cell lung cancer (NSCLC). Ann Oncol 2005;16 Suppl 1:i28-9.

6. Morgensztern D, Campo MJ, Dahlberg SE, Doebele RC, Garon E, Gerber DE, et al. Molecularly Targeted Therapies in Non Small Cell Lung Cancer Annual Opdate 2014. J Thorac Oncol 2015; 10(101): S1-63.

7. Deniz G, van de Veen W, Akdis M. Natural killer cells in patients with allergic diseases. J Allergy Clin Immunol 2013;132(3):527-35.

8. Platonova S, Cherfils-Vicini J, Damotte D, Crozet L, Vieillard V, Validire P.Profound coordinated alterations of intratumoral NK cell phenotype and function in lung carcinoma. Cancer Res 2011;71(16):5412-22.

9. Al Omar SY, Marshall E, Middleton D, Christmas SE. Increased killer immunoglobulin-like receptor expression and functional defects in natural killer cells in lung cancer. Immunology 2011;133(1):94-104.

10. Li Yang, Liping Wang, and Yi Zhang. Immunotherapy for lung cancer: advances and prospects. Am J Clin Exp Immunol 2016;5(1):1-20.

11. Villegas FR, Coca S, Villarrubia VG, Jime'nez R, Chillo'n MJ, et al. Prognostic significance of tumor infiltrating natural killer cells subset CD57 inpatients with squamous cell lung cancer. Lung Cancer 2002;35:23-28.

12. Ferlazzo G, Thomas D, Lin SL, Goodman K, Morandi B, Muller WA, Moretta A, Münz C. The abundant NK cells in human secondary lymphoid tissues require activation to express killer cell Ig-like receptors and become cytolytic. J Immunol 2004;172(3):1455-62.

13. Jonges $L E$, Albertsson $P$, van Vlierberghe RL, Ensink NG, Johansson $B R$, van de Velde $C J$, et al. The phenotypic heterogeneity of human natural killer cells: presence of at least 48 different subsets in the peripheral blood. Scand J Immunol2001;53:103-10.

14. Cooper MA, Fehniger TA, Caligiuri MA. The biology of human natural killer-cell subsets. Trends Immunol 2001;22:633-40.

15. Poli A, Michel T, Theresine M, Andres E, Hentges F, Zimmer J. CD56bright natural killer (NK) cells: an important NK cell subset. Immunology 2009;126:458-65.

16. Vivier E, Raulet DH, Moretta A, Caligiuri MA, Zitvogel L, Lanier LL, Yokoyama WM, Ugolini S. Innate or adaptive immunity? The example of natural killer cells. Science 2011;331:44.

17. Marcus A, Gowen BG, Thompson TW, lannello A, Ardolino M, Deng W, Wang L Shifrin N, Raulet $\mathrm{DH}$. Recognition of tumors by the innate immune system and natural killer cells. Adv Immunol 2014;122:91-128.

18. Peter ME, Krammer PH. The CD95(APO-1/Fas) DISC and beyond. Cell Death Differ 2003; 10(1):26-35.

19. Bauer, S. et al. Activation of natural killer cells and T cells by NKG2D, a receptor for stressinducible MICA. Science 1999;285:727-730.

20. Pende D, Cantoni C, Rivera P, Vitale M, Castriconi R, Marcenaro S, Nanni M, Biassoni R, Bottino C, Moretta A, Moretta L. Role of NKG2D in tumor cell lysis mediated by human NK cells: cooperation with natural cytotoxicity receptors and capability of recognizing tumors of nonepithelial origin. Eur J Immunol 2001;31(4):1076-86.

21. Sopori M. Effects of cigarette smoke on the immune system. Nat Rev Immunol 2002;2(5):3727.

22. Lu LM, Zavitz CC, Chen B, Kianpour S, Wan Y, Stämpfli MR. Cigarette Smoke Impairs NK CellDependent Tumor Immune Surveillance. J Immunol 2007;178(2):936-43. 
23. Motz GT, Eppert BL, Wortham BW, Amos-Kroohs RM, Flury JL, Wesselkamper SC, Borchers MT. Chronic Cigarette Smoke Exposure Primes NK Cell Activation in a Mouse Model of Chronic Obstructive Pulmonary Disease. J Immunol. 2010;184:4460-4469.

24. Sopori ML, Gairola CC, DeLucia AJ, Bryant LR, Cherian S. Immune responsiveness of monkeys exposed chronically to cigarette smoke Clin Immunol Immunopathol 1985;36(3):338.

25. Mian MF, Lauzon NM, Stampfli MR, Mossman KL, Ashkar AA. Impairment of human NK cell cytotoxic activity and cytokine release by cigarette smoke. J Leukoc Biol 2008;83:74-784.

26. Mian MF, Pek EA, Mossman KL, Stampfli MR, Ashkar AA. Exposure to cigarette smoke suppresses IL-15 generation and its regulatory NK cell functions in poly I:C-augmented human PBMCs. Mol Immunol 2009;46:3108-3116.

27. Mian MF, Steampfli MR, Mossman KL, Ashkar AA. Cigarette smoke attenuation of poly I: Cinduced innate antiviral responses in human PBMC is mainly due to inhibition of IFNbeta production. Mol Immunol 2009;46:825e9.

28. Mian MF, Pek EA, Mossman KL, St€ampfli MR, Ashkar AA. Exposure to cigarette smoke suppresses IL-15 generation and its regulatory NK cell functions in poly I: C-augmented human PBMCs. Mol Immunol 2009;46:3108e16.

29. Moszczyński P, Zabiński Z, Moszczyński P Jr, Rutowski J, Słowiński S, Tabarowski Z. Immunological findings in cigarette smokers. Toxicol Lett 2001;118(3):121-7.

30. Andreoli C, Bassi A, Gregg EO, Nunziata A, Puntoni R, Corsini E. Effects of cigarette smoking on circulating leukocytes and plasma cytokines in monozygotic twins. Clin Chem Lab Med 2015;53(1):57-64.

31. Luppi $P$, Lain KY, Jeyabalan A, DeLoia JA. The effects of cigarette smoking on circulating maternal leukocytes during pregnancy. Clin Immunol 2007;122(2):214-9.

32. Hodge G, Barnawi J, Jurisevic C, Moffat D, Holmes M, Reynolds PN, Jersmann H, Hodge S. Lung cancer is associated with decreased expression of perforin, granzyme B and interferon (IFN)- $\gamma$ by infiltrating lung tissue T cells, natural killer (NK) T-like and NK cells. Clin Exp Immunol 2014;178(1):79-85.

33. Castriconi R, Cantoni C, Della Chiesa M, Vitale M, Marcenaro E, Conte R, Biassoni R, Bottino C, Moretta L, Moretta A. Transforming growth factor beta 1 inhibits expression of NKp30 and NKG2D receptors: consequences for the NK-mediated killing of dendritic cells. Proc Natl Acad Sci U S A 2003;100(7):4120-5.

34. Huang AL, Liu SG, Qi WJ, Zhao YF, Li YM, Lei B, Sheng WJ, Shen H. TGF- $\beta 1$ protein expression in non-small cell lung cancers is correlated with prognosis. Asian Pac J Cancer Prev 2014;15(19):8143-7.

35. De Vita F, Orditura M, Galizia G, Romano C, Roscigno A, Lieto E, Catalano G. Serum interleukin10 levels as a prognostic factor in advanced non-small cell lung cancer patients. Chest 2000;117:365-73.

36. Hatanaka H, Abe Y, Kamiya T, Morino F, Nagata J, Tokunaga T, Oshika Y, Suemizu H, Kijima H, Tsuchida T, Yamazaki $H$, Inoue $H$, et al. Clinical implications of interleukin-10 induced by nonsmall-cell lung cancer. Ann Oncol 2000;11:815-819.

37. Schneider T, Kimpfler S, Warth A, Schnabel PA, Dienemann H, Schadendorf D, Hoffmann H, Umansky $\mathrm{V}$. Foxp3(+) regulatory $T$ cells and natural killer cells distinctly infiltrate primary tumors and draining lymph nodes in pulmonary adenocarcinoma. J Thorac Oncol 2011;6(3):432-438.

38. Ndhlovu LC, Lopez-Vergès S, Barbour JD, Jones RB, Jha AR, Long BR, Schoeffler EC, Fujita $T$, Nixon DF, Lanier LL. Tim-3 marks human natural killer cell maturation and suppresses cellmediated cytotoxicity. Blood 2012;119(16):3734-43.

39. Xu L, Huang Y, Tan L, Yu W, Chen D, Lu C, He J, Wu G, Liu X, Zhang Y. Increased Tim-3 expression in peripheral NK cells predicts a poorer prognosis and Tim-3 blockade improves NK cell- 
mediated cytotoxicity in human lung adenocarcinoma. Int Immunopharmacol 2015;29(2):635641.

40. Fend L, Rusakiewicz S, Adam J, Bastien B, Caignard A, Messaoudene M, et al. Prognostic impact of the expression of NCR1 and NCR3 NK cell receptors and PD-L1 on advanced non-small cell lung cancer. Oncoimmunology 2016;6(1):e1163456.

41. Jin S, Deng Y, Hao JW, Li Y, Liu B, Yu Y, Shi FD, Zhou QH. NK cell phenotypic modulation in lung cancer environment. PLoS One 2014;9(10):e109976.

42. Carrega P1, Morandi B, Costa R, Frumento G, Forte G, Altavilla G, Ratto GB, Mingari MC, Moretta L, Ferlazzo G. Natural killer cells infiltrating human nonsmall-cell lung cancer are enriched in CD56 bright CD16(-) cells and display an impaired capability to kill tumor cells. Cancer 2008;112(4):863-75.

43. Esendagli G, Bruderek K, Goldmann T, Busche A, Branscheid D, Vollmer E, Brandau S. Malignant and non-malignant lung tissue areas are differentially populated by natural killer cells and regulatory T cells in non-small cell lung cancer. Lung Cancer 2008;59(1):32-40.

44. Lowry LE, Zehring WA. Potentiation of Natural Killer Cells for Cancer Immunotherapy: A Review of Literature. Front Immunol 2017;8:1061.

45. Loza, M. J. \& Perussia, B. The IL- 12 signature:NK cell terminal CD56+high stage and effector functions. J. Immunol2004;172: 88-96.

46. Romee, R. et al. NK cell CD16 surface expression and function is regulated by a disintegrin and metalloprotease- 17 (ADAM17). Blood 2013;121:3599-3608.

47. Boutet, $P$. et al. Cutting edge: the metalloproteinase ADAM17/TNF- $\alpha$-converting enzyme regulates proteolytic shedding of the MHC class I-related chain B protein. J. Immunol 2009;182: 49-53.

48. Luna JI1, Grossenbacher SK1, Murphy WJ1,2, Canter RJ3. Targeting Cancer Stem Cells with Natural Killer Cell Immunotherapy. Expert Opin Biol Ther 2017;17(3):313-324.

49. Kim S, lizuka K, Aguila HL, et al. In vivo natural killer cell activities revealed by natural killer celldeficient mice. Proceedings of the National Academy of Sciences of the United States of America 2000;97(6):2731-2736.

50. Tonn T, Schwabe D, Klingemann HG, et al. Treatment of patients with advanced cancer with the natural killer cell line NK-92. Cytotherapy 2013;15(12):1563-1570.

51. Hiraki A, Kiura K, Yamane H, Nogami N, Tabata M, Takigawa N, Ueoka H, Tanimoto M, Harada M. Interleukin-12 augments cytolytic activity of peripheral blood mononuclear cells against autologous lung cancer cells in combination with IL-2. Lung Cancer. 2002;35(3):329-333.

52. Miller JS, Morishima C, McNeel DG, Patel MR, Kohrt HE, Thompson JA, et al. A First-in-Human Phase 1 Study of Subcutaneous Outpatient Recombinant Human IL-15 (rhIL-15) in Adults with Advanced Solid Tumors. Clin Cancer Res 2017 Dec 4. pii: clincanres.2451.2017

53. Cui F, Ji J, Lv H, Qu D, Yu C, Yang Y, Xu Y. Immune responsiveness in a mouse model of combined adoptive immunotherapy with NK and dendritic cellsJ Cancer Res Ther 2013;9 Suppl:S162-168.

54. Zhang G, Zhao H, Wu J, Li J, Xiang Y, Wang G, Wu L, Jiao S. Adoptive immunotherapy for nonsmall cell lung cancer by NK and cytotoxic $T$ lymphocytes mixed effector cells: retrospective clinical observation. Int Immunopharmacol 2014;21(2):396-405.

55. Lin M, Liang SZ, Wang XH et al. Clinical efficacy of percutaneous cryoablation combined with allogenic NK cell immunotherapy for advanced non-small cell lung cancer. Immunol Res 2017;65(4):880-887.

56. Krause SW, Gastpar R, Andreesen R, Gross C, Ullrich H, Thonigs G, et al. Treatment of Colon and Lung Cancer Patients with ex Vivo Heat Shock Protein 70-Peptide-Activated, Autologous Natural Killer Cells : A Clinical Phase I Trial. Clin Cancer Res 2004;10:3699-3707 
57. Specht HM, Pelzel J, Hautmann H, Huber RM, Schossow B, Molls M, Multhoff G. P67. Targeted natural killer (NK) cell based adoptive immunotherapy for the treatment of patients with nonsmall cell lung cancer (NSCLC) after radiochemotherapy (RCT) - clinical application of NK cells activated by heat shock protein 70 (Hsp70). J Immunother Cancer 2014;2(Suppl 2):P41.

58. Ding X, Cao H, Chen X, Jin H, Liu Z, Wang G, Cai L, Li D, Niu C, Tian H, Yang L, Zhao Y, Li W, Cui J. Cellular immunotherapy as maintenance therapy prolongs the survival of the patients with small cell lung cancer. J Transl Med 2015;13:158. 